\title{
Calculation of the power of an iris clip lens for distant vision
}

\author{
M. G. GOLENBRANDER \\ Department of Ophthalmology, University of Leyden, The Netherlands
}

Until now most eyes treated with a clip lens have had inserted a device of the following specification:

$\begin{array}{ll}\text { Diameter } & 4.8 \mathrm{~mm} . \\ \text { Thickness }(t) & 0 \cdot 65-0.75 \mathrm{~mm} . \\ \text { Radius of anterior surface } & 7.95 \mathrm{~mm} . \\ \text { Radius of posterior surface } & \text { plane } \\ \text { Refractive index }\left(n_{2}\right) & \mathbf{I} \cdot 49 \mathbf{I} \\ \text { Refractive power in water } & 20 \cdot 5 \text { diopters }\end{array}$

It is however feasible to adapt the power of the lens to each individual. This can be done by calculation, using the following data:

(I) The refractive index of aqueous and vitreous $\left(n_{1}\right)=\mathrm{I} \cdot 336$

(2) The refractive power of the cornea $\left(\mathrm{F}_{\mathrm{C}}\right)$ as read from the ophthalmometer of Javal.

(3) The depth of the anterior chamber $(v)$. By this we mean the distance between the anterior surface of the cornea and the anterior surface of the clip lens at the optical axis, strictly speaking the thickness of the cornea plus the depth of the anterior chamber.

(4) The axial length of the eye $(l)$. This is the distance from the anterior surface of the cornea to the fovea centralis. The distance from the anterior surface of the cornea to the anterior surface of the lens can be measured accurately by means of the Goldmann apparatus on the slit lamp and the distance from the anterior surface of the lens to the anterior surface of the retina can be measured with sufficient exactitude by ultrasonography. For the thickness of the retina, $0.5 \mathrm{~mm}$. must be added.

In addition we must know the position of the principal points of the cornea $(a)$ and of the clip lens $(b)$. (Fig. I overleaf):

(a) The cornea itself acts as a thin lens, because the posterior surface is a little more curved than the anterior surface. For the position of the equivalent cornea I adopt, according to Gullstrand, a point $0.05 \mathrm{~mm}$. in front of the vertex corneae. In the calculation, this small distance has been added to the measured depth of the anterior chamber.

(b) The clip lens $\left(\mathrm{F}_{\mathrm{L}}\right)$, consisting of an anterior surface with the refracting power $\mathbf{F}_{\mathbf{L}_{1}}$, a thickness $t$, and a plane posterior surface with no refracting power $\left(\mathrm{F}_{\mathrm{L}_{2}}=0\right)$. The position of the second principal point $\left(\mathrm{P}_{\mathrm{L}}^{\prime}\right)$ of the lens with respect to the position of the second principal point of the posterior surface $\left(\mathrm{P}^{\prime} \mathrm{I}_{2}\right)$ is given by the equation:

$\mathrm{P}_{\mathrm{L}_{2}}^{\prime} \mathrm{P}_{\mathrm{L}}^{\prime}=-\frac{n_{1}}{n_{2}} t \frac{\mathrm{F}_{\mathrm{L}_{1}}}{\mathrm{~F}_{\mathrm{L}}}=-0.895 t$ 


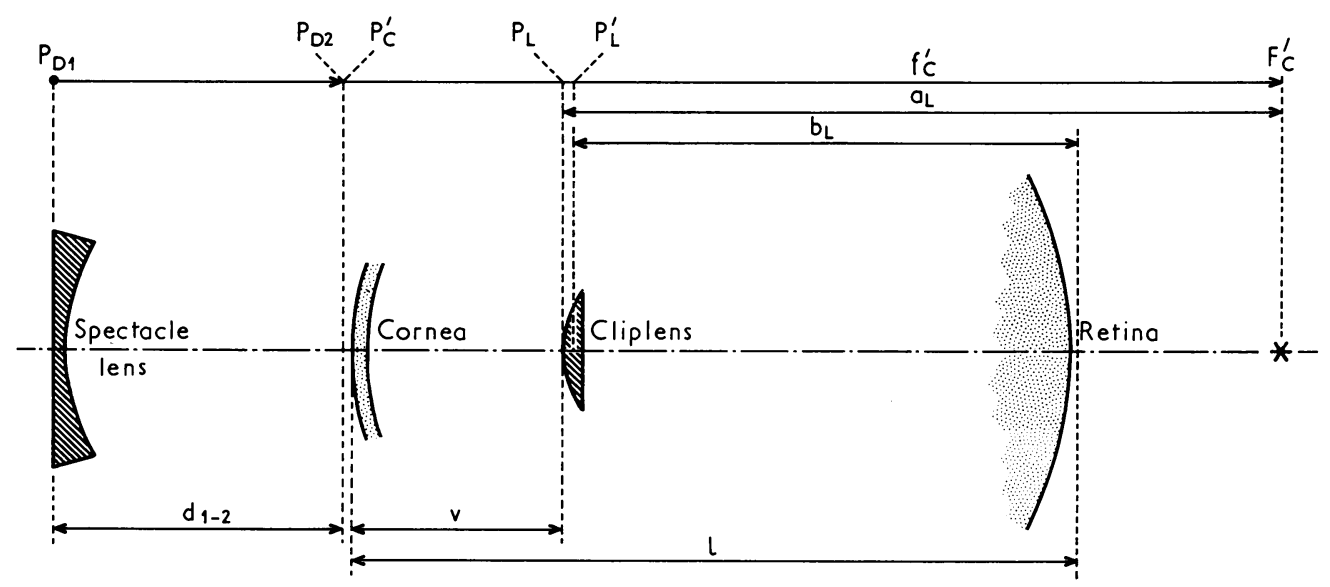

FIG. I. Position of the optical points used in the calculations

The distance of the second principal point of the clip lens from the anterior surface of the lens is consequently $\left(t^{\prime}-0.895\right) t=0.105 t$, or about $0.05 \mathrm{~mm}$. (0.00005 m.).

The calculation of the power of the clip lens is as follows:

For the refractive power of the cornea $\left(\mathrm{F}_{\mathbf{C}}\right)$ the equation:

$\mathrm{F}_{\mathrm{C}}=\frac{n_{1}}{f^{\prime} \mathrm{C}}$ or $f^{\prime}{ }_{\mathrm{C}}=\frac{n_{1}}{\mathrm{~F}_{\mathrm{C}}}$

For the refraction of the clip lens $\left(\mathrm{F}_{\mathrm{L}}\right)$ the equation:

$\mathrm{F}_{\mathrm{L}}=\mathrm{B}_{\mathrm{L}}-\mathrm{A}_{\mathrm{L}}=\frac{n_{1}}{b_{\mathrm{L}}}-\frac{n_{1}}{a_{\mathrm{L}}}$

where $b_{\mathrm{L}}=-v-0 \cdot 00005$,

$a_{\mathrm{L}}=f^{\prime}{ }_{\mathrm{C}}-v-0 \cdot 00005$, and $f^{\prime}{ }_{\mathrm{C}}=\frac{n_{1}}{\mathrm{~F}_{\mathrm{C}}}$

Hence, $\mathrm{F}_{\mathrm{L}}=\frac{n_{1}}{l-v-0 \cdot 00005}-\frac{n_{1}}{\frac{n_{1}}{\mathrm{~F}_{\mathrm{C}}}-v-0 \cdot 00005}$

The last equation shows that the length of the eye $(l)$ and the refractive power of the cornea $\left(\mathrm{F}_{\mathrm{C}}\right)$ occur only once in the formula and each in a separate part. Their variations N do not influence each other and can simply be added.

This is not so for the depth of the anterior chamber $(v)$. But fortunately its influence is $\mathrm{N}$ not so great, for which reason it can be treated as an independently acting variable.

For practical use it is adequate to start from a normal eye with a refractive power of the cornea $\left(\mathrm{F}_{\mathrm{C}}\right)$ of 42 diopters, an anterior chamber $(v)$ of $3.5 \mathrm{~mm}$. , and an axial length $(l) \stackrel{\mathbb{\Phi}}{\rightarrow}$ of $24 \mathrm{~mm}$. Such an eye requires an artificial lens $\left(F_{L}\right)$ of 18 diopters. From this point of 0 departure it is simple to calculate the influence of variations of the cornea $\left(\mathbf{F}_{\mathbf{C}}\right)$ only, of the 


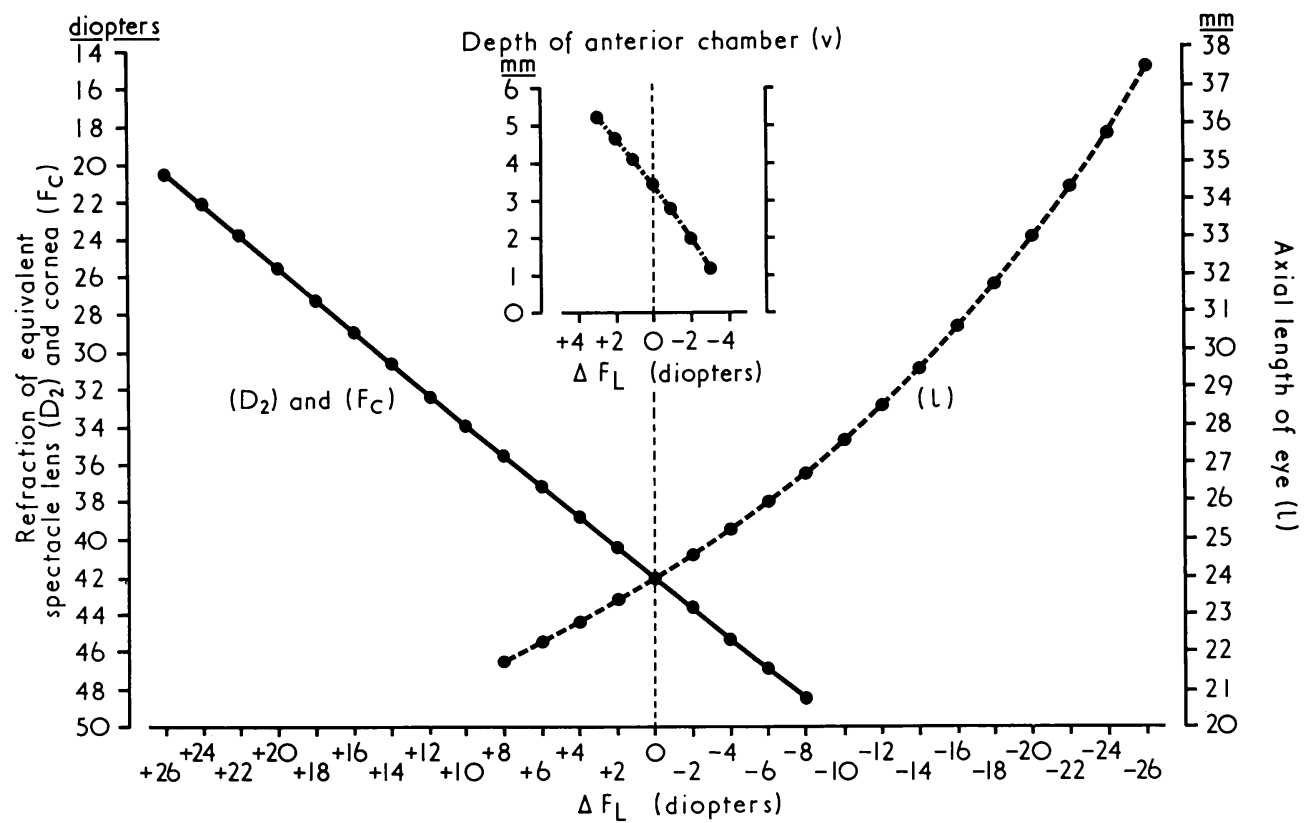

F I G. 2. Deflection $\left(\Delta \mathrm{F}_{\mathrm{L}}\right)$ from normal ( $\mathrm{I} 8$ dptr) of the refractive power of the clip lens as a result of the refractive power of the spectacle lens $(D)+$ cornea $(\mathrm{Fc})$, depth of anterior chamber $(v)$, and axial length of eye $(l)$

anterior chamber $(v)$ only, and of the axial length $(l)$ only. The influence of a combination of variations is thus a matter of simple addition.

In this way we can arrive at the power of the clip lens, giving a sharp image on the retina without the use of spectacles. But in many cases, especially when the patient was accustomed to wearing strong spectacles before the operation, this is not the most advisable solution. When the patient needs no glass for the operated eye but does need one for the fellow eye, he is apt to complain of aniseikonia. If he did not suffer from aniseikonia before the operation, the best solution seems to give him a clip lens of such a power that with spectacles his retinal images are as great as before.

The position of the clip lens is somewhat more anterior than the position of the natural lens and this works out to a greater image on the retina.

In the schematic eye of Gullstrand, an anterior shift of $\mathrm{I} \mathrm{mm}$. of the lens increases the refractive power of the eye from $5^{8 \cdot 6}$ I to $59 \cdot 2$ I diopters, and displaces the second principal point of the eye $0.50 \mathrm{~mm}$. towards the cornea and the second nodal point $0.34 \mathrm{~mm}$; this enlarges the retinal image $2 \cdot 0$ per cent.

The main purpose of using calculation for a personal clip lens is not to make a spectacle lens superfluous, but to avoid aniseikonia.

To cope with this aniseikonia, one has to select a slightly stronger clip lens, and accordingly the spectacle lens (if positive) has to be a little weaker, so that this combination acts like a reversed Dutch telescope. A negative lens of 0.98 diopters and a positive lens of I diopter placed $2 \mathrm{~cm}$. behind the former reduces the image by 2 per cent.

Assuming that the optical centre of the clip lens lies I $\mathrm{mm}$. anterior to the optical centre of the natural eye lens, one has to select a clip lens I diopter stronger to get rid of this aniseikonia. 
But there is still another consideration. The wearing of strong spectacles lenses, either positive or negative, has difficulties of its own, and this leads to the trend to reduce the power of the spectacle lens if possible. Now it is a fact that a change of I diopter in the spectacle lens does not lead to complaints of aniseikonia. In consequence, there is no objection to reducing the power of the future spectacle lens by I diopter and to changing the power of the clip lens accordingly. This means, in the case of positive spectacles, an increase of I diopter in the power of the clip lens, and in the case of negative spectacles a decrease of I diopter in the power of the clip lens.

Because a clip lens has no accommodation, persons with adequate accommodation in the other eye, need a lens for an intermediate distance. This means adding another diopter of extra power to the clip lens for young persons.

These two considerations together lead to the following rules:

(I) If the patient wears a positive correction before operation, increase the power of the $\frac{D}{0}$. clip lens by 2 diopters.

(2) If the patient wears no spectacles, increase the power of the clip lens by I diopter.

(3) If the patient wears a negative correction, do not change the calculated power of the clip lens.

(4) For young persons add another diopter to the clip lens.

The simplest way to cope with spectacle lenses is to convert their power $\left(D_{1}\right)$ at the position in the spectacle frame, i.e. at a distance of $d_{1-2}$ in front of the principal point of the cornea, to an equivalent power $\left(D_{2}\right)$ at the position of the principal point of the cornea and $\vec{\varphi}$ then to take $\mathrm{D}_{2}$ and $\mathrm{F}_{\mathrm{C}}$ together.

$D_{1}$ and $D_{2}$ must have their focal points in common.

Consequently $f_{1}-d_{1-2}=f_{2}$.

One has to bear in mind that spectacle glasses, especially negative glasses, are seldom worn at the place of the first focal point, but nearer to the eye. Hence its place should be measured.

The computation leads to the following results:

\begin{tabular}{|c|c|c|c|c|c|c|c|c|}
\hline \multirow{2}{*}{\multicolumn{9}{|c|}{$\begin{array}{c}\qquad \mathrm{D}_{1} \\
\text { is changed into: }\end{array}$}} \\
\hline & & & & & & & & \\
\hline$d_{1-2}=$ Io $\mathrm{mm}$ & & $+\mathbf{I I} \cdot \mathbf{I}$ & $+8 \cdot 7$ & $+6 \cdot 4$ & $-5 \cdot 7$ & $-7 \cdot 4$ & $-9 \cdot \mathbf{I}$ & D \\
\hline$d_{1-2}=12 \mathrm{~mm}$ & & $+11 \cdot 4$ & $+8 \cdot 85$ & $+6 \cdot 5$ & $-5 \cdot 6$ & $-7 \cdot 3$ & $-8 \cdot 9$ & D \\
\hline$d_{1-2}=14 \mathrm{~mm}$ & & $+11 \cdot 6$ & $+9 \cdot 0$ & $+6 \cdot 55$ & $-5 \cdot 55$ & $-7 \cdot 2$ & $-8 \cdot 8$ & $\mathrm{D}$ \\
\hline$d_{1-2}=\mathrm{I} 6 \mathrm{~mm}$ & & $+\mathrm{II} \cdot 9$ & $+9 \cdot 2$ & $+6 \cdot 65$ & $-5 \cdot 5$ & $-7 \cdot 1$ & $-8 \cdot 6$ & $\mathrm{D}$ \\
\hline$D_{1}$ & -12 & -14 & -16 & -18 & -20 & -22 & -24 & D \\
\hline anged into: & & & & & & & & \\
\hline $\begin{array}{l}d_{1}-2=10 \mathrm{~mm} \\
d_{1-2}=12 \mathrm{~mm} .\end{array}$ & $-10 \cdot 7$ & $-12 \cdot 3$ & $-13 \cdot 8$ & -15.3 & $-16 \cdot 7$ & $-18 \cdot 0$ & $-19 \cdot 3$ & D \\
\hline $\begin{array}{l}d_{1}-2=12 \mathrm{~mm} \\
d_{1-2}=14 \mathrm{~mm}\end{array}$ & $-10 \cdot 5$ & $-12 \cdot 0$ & -13.4 & $-14 \cdot 8$ & $-16 \cdot 1$ & $-17 \cdot 4$ & $-18 \cdot 6$ & D \\
\hline $\begin{array}{l}-2=14 \mathrm{~mm} \\
-2=16 \mathrm{~mm} .\end{array}$ & $-10 \cdot 25$ & $-11 \cdot 7$ & $-13 \cdot 1$ & -14.4 & $-15 \cdot 6$ & $-16 \cdot 8$ & $-18 \cdot 0$ & D \\
\hline$-2=16 \mathrm{~mm}$ & $-10 \cdot 0$ & $-11 \cdot 4$ & $-12 \cdot 75$ & $-14 \cdot 0$ & $-15 \cdot 1$ & $-16 \cdot 25$ & $-17 \cdot 3$ & D \\
\hline
\end{tabular}

For weak lenses, just as for little shifts, the equation $\Delta \mathrm{D}=d_{1-2} \mathrm{D}^{2}$ applies.

The results of the calculations for the power of the clip lens are as follows. They are calculated in the reverse direction, beginning with equal variations for $F_{L}$ in order to put 
all the results together. Only for the depth of the anterior chamber is the calculation given in full.

\begin{tabular}{|c|c|c|c|c|c|c|c|c|}
\hline \multirow{2}{*}{$\begin{aligned} v & = \\
\mathbf{F}_{\mathrm{C}} & =4^{2} \cdot 0\end{aligned}$} & $5 \cdot 2$ & $4 \cdot 7$ & $4 \cdot 1$ & $3 \cdot 5$ & $2 \cdot 8$ & $2 \cdot 0$ & $\mathrm{I} \cdot 2$ & $\mathrm{~mm}$. \\
\hline & & & & & & & & \\
\hline$f_{\mathrm{C}}^{\prime}=3^{\mathrm{I}} \cdot 8$ & & & & & & & & \\
\hline $\mathrm{c}-v-0 \cdot 05=a_{\mathrm{L}}$ & $26 \cdot 55$ & $27 \cdot 05$ & $27 \cdot 65$ & $28 \cdot 25$ & $28 \cdot 95$ & $29 \cdot 75$ & $30 \cdot 55$ & $\mathrm{~mm}$. \\
\hline$\frac{n}{a_{\mathrm{L}}}=\mathrm{A}_{\mathrm{L}}$ & $50 \cdot 4$ & $49 \cdot 5$ & $48 \cdot 3$ & $47 \cdot 3$ & $46 \cdot 2$ & $45^{\circ} \mathrm{O}$ & $43 \cdot 8$ & dptr \\
\hline$l=24$ & & & & & & & & \\
\hline$l-v-0.05=b_{\mathrm{L}}$ & $18 \cdot 75$ & $19 \cdot 25$ & $19 \cdot 85$ & $20 \cdot 45$ & $21 \cdot 15$ & $21 \cdot 95$ & $22 \cdot 75$ & $\mathrm{~mm}$. \\
\hline$\frac{n}{b_{\mathrm{L}}}=\mathrm{B}_{\mathrm{L}}$ & $71 \cdot 4$ & $69 \cdot 5$ & $67 \cdot 3$ & $65 \cdot 3$ & $63 \cdot 2$ & $6 \mathrm{I} \cdot 0$ & $58 \cdot 8$ & \\
\hline $\mathrm{B}_{\mathrm{L}}-\mathrm{A}_{\mathrm{L}}+\mathbf{F}_{\mathbf{L}}$ & 21 & 20 & 19 & 18 & 17 & 16 & 15 & dptr \\
\hline $\mathrm{F}_{\mathrm{L}}-\mathrm{I} 8=\Delta \mathrm{F}_{\mathrm{L}}$ & +3 & +2 & $+\mathbf{I}$ & o & $-\mathbf{I}$ & -2 & -3 & dptr \\
\hline
\end{tabular}

In the same manner the influence of the refraction of the cornea $\left(\mathbf{F}_{\mathbf{C}}\right)$ and the equivalent spectacle lens $\left(D_{2}\right)$ on the deflection $\left(\Delta F_{L}\right)$ of the power of the clip lens, with a standard depth of the anterior chamber $(v=3.5 \mathrm{~mm}$.) and a standard axial length $(l=24 \mathrm{~mm}$.) can be calculated.

The same holds true for the axial length $(l)$, with a standard power of the cornea $\left(\mathbf{F}_{\mathbf{C}}\right)$ and the equivalent spectacle lens $\left(\mathrm{D}_{2}\right)$ and a standard depth of the anterior chamber $(v=3.5 \mathrm{~mm}$.).

The results may be tabulated as follows:

\begin{tabular}{|c|c|c|c|}
\hline$F_{C}+D_{\mathbf{2}}$ (diopters) & $\triangle \mathbf{F}_{\mathrm{L}}$ (diopters) & $\triangle \mathrm{F}_{\mathrm{L}}($ diopters $)$ & $l(\mathrm{~mm})$. \\
\hline $20 \cdot 2$ & +26 & -26 & $37 \cdot 5$ \\
\hline $22 \cdot 0$ & +24 & -24 & $35 \cdot 8$ \\
\hline $23 \cdot 7$ & +22 & -22 & $34 \cdot 3$ \\
\hline $25 \cdot 4$ & +20 & -20 & $33^{\circ} 0$ \\
\hline $27 \cdot 2$ & +18 & -18 & $3 \mathrm{I} \cdot 8$ \\
\hline $28 \cdot 9$ & +16 & -16 & $30 \cdot 7$ \\
\hline $30 \cdot 6$ & +14 & -14 & $29 \cdot 6$ \\
\hline $32 \cdot 3$ & +12 & -12 & $28 \cdot 6$ \\
\hline $34 \cdot 0$ & +10 & -10 & $27 \cdot 7$ \\
\hline $35^{\cdot} 6$ & +8 & -8 & $26 \cdot 8$ \\
\hline $37 \cdot 2$ & +6 & -6 & $26 \cdot 0$ \\
\hline $38 \cdot 8$ & +4 & -4 & $25 \cdot 3$ \\
\hline $4^{0} \cdot 4$ & +2 & -2 & $24 \cdot 6$ \\
\hline $4^{2} \cdot 0$ & o & 0 & $24 \cdot 0$ \\
\hline $43 \cdot 6$ & -2 & +2 & $23 \cdot 4$ \\
\hline $4 \dot{5} \cdot 2$ & -4 & +4 & $22 \cdot 8$ \\
\hline $46 \cdot 7$ & -6 & +6 & $22 \cdot 3$ \\
\hline $48 \cdot 3$ & -8 & +8 & $2 \mathrm{I} \cdot 8$ \\
\hline
\end{tabular}

Some examples may help in handling these figures*.

* The author will be pleased to give advice in any special case 
Example I A young man, not wearing spectacles, has a corneal refraction of $43 \mathrm{dptr}\left(\Delta \mathrm{F}_{\mathrm{L}}=-\mathrm{I}\right)$, a depth of anterior chamber of $4 \cdot 5 \mathrm{~mm} .\left(\Delta \mathrm{F}_{\mathrm{L}}=+\mathrm{I} \cdot 5\right)$, and an axial length of $24 \cdot 6 \mathrm{~mm} .\left(\Delta \mathrm{F}_{\mathrm{L}}=+\mathrm{I}\right)$. For sharp vision at medium distance $\left(\Delta \mathbf{F}_{\mathbf{L}}=+\mathbf{I}\right)$ he needs a clip lens of $\mathbf{I} 8-\mathbf{I}+\mathbf{I} \cdot 5+\mathbf{I}+\mathbf{I}=$ $20 \cdot 5$ dptr.

Example 2 A man wearing spectacles of $+6 \mathrm{dptr}$ at $\mathrm{I} \cdot 5 \mathrm{~mm}$. in front of the cornea $\left(\mathrm{D}_{2}=+6 \cdot 5\right)$, a corneal refraction of $39 \mathrm{dptr}$, together $45.5 \mathrm{dptr}\left(\Delta \mathrm{F}_{\mathrm{L}}=-4\right)$, has an anterior chamber depth of $3.5 \mathrm{~mm} .\left(\Delta \mathrm{F}_{\mathrm{L}}=0\right)$, and an axial length of $23 \mathrm{~mm} .\left(\Delta \mathrm{F}_{\mathrm{L}}=+3\right)$. For vision at distance he needs a clip lens of $18-4+3+0+2=19$ dptr.

Example 3 A young man wearing spectacles of $-20 \mathrm{dptr}$ at $10 \mathrm{~mm}$. in front of the cornea $\left(\mathrm{D}_{2}=\right.$ I6.7), a corneal refraction of $46 \cdot 7 \mathrm{dptr}$, together $30 \mathrm{dptr}\left(\Delta \mathrm{F}_{\mathrm{L}}=+14 \cdot 5\right)$, has an anterior chamber depth of $4 \mathrm{~mm} .\left(\Delta \mathrm{F}_{\mathrm{L}}=+0.5\right)$, and an axial length of $33 \mathrm{~mm} .\left(\Delta \mathrm{F}_{\mathrm{L}}=-20\right)$. For sharp vision at medium distance he needs a clip lens of $18+14 \cdot 5+0.5-20+1+0=14$ dptr.

\section{Summary}

The required power of an iris clip lens as a substitute for the extracted eye-lens is dependent on the refraction of the cornea, the depth of the anterior chamber, the axial length of the eye, and (to avoid aniseikonia) the refraction of the fellow eye.

The required power can be calculated within reasonable limits of accuracy.

This paper describes a method of accomplishing this and offers the results in the form of $\bigcirc$ Tables. 